The first half of the book is devoted to a fairly comprehensive description of the various experimental techniques which have been developed for studying fast reactions. A brief outline of flow methods is followed by a discussion of large perturbation methods including flash photolysis, pulse radiolysis and shock tube methods. A nanosecond laser photolysis system is described but there is no mention of picosecond lasers. Chemical relaxation methods which utilize a single small perturbing pulse are dealt with in some detail but the treatment of those based on the use of periodic disturbances is much briefer. The author's treatment of competition methods based on nuclear magnetic resonance, electron spin resonance, fluorescence and polarographic measurements which are covered in less than twenty pages requires a great deal of background knowledge which even industrious senior undergraduates are likely to find somewhat exacting. However, research workers in other areas of chemistry may find the concise treatment to their liking.

The second half of the book is devoted to selected examples of reactions which have been studied by rapid reaction techniques. In the third chapter proton transfer reactions and the reactions of the hydrated electron are discussed in some detail and several useful tables of elementary rate constants are given. This chapter concludes with a résumé of recent results and theories concerning metal complex formation. The final chapter gives some indication of advances concerning rapid reactions in biological systems with considerable emphasis on enzyme reactions together with comments on the role of metals and nucleic acids in biology. At the end of each chapter a short list of useful references is given; in addition there are some problems on the second and third chapters.

The book is well written and produced. One doubts, however, if it is likely to be preferred to previous textbooks which act as an introduction to the study of the kinetics of fast chemical reactions. In spite of this, most libraries will probably want to buy this concise and thoroughly readable book.

F. WILKINSON

\section{Inorganic Reactions}

Inorganic Reaction Mechanisms. Vol. 1. Senior Reporter J. Burgess. Pp. xv+ 338. (The Chemical Society: London, June 1971.) $£ 7$.

SURVEYS of recent work are a valuable activity, of long standing, within the Chemical Society. The annual reports contained articles which, broadly speaking, fell into two classes. There were comprehensive reviews, covering all work in a given area. Much rarer were occasional masterpieces in which selection and critical evaluation of recent trends were keynotes.

This inaugural specialist report on mechanisms of inorganic reactions is of the former kind. It contains a full though rather uncritical survey of relevant literature from January 1969 to August 1970, with no obvious omissions. There are extensive tabulations, particularly in the sections on oxidation.

Some errors have been missed by the proof readers. This is not too distressing because the production of these volumes is gratifyingly rapid. There are, however, several perpetuations of error, which are deplorable in a publication of the Chemical Society--nomenclature for optically active compounds is antiquated; the effect of acid on the aquation of halo-complexes is called, very inexactly, catalysis.

Many opportunities to resolve controversial points or to help generalizations to emerge have been burked. For example, the bare statement is made about $\left[\mathrm{Co}\left(\mathrm{C}_{2} \mathrm{O}_{4}\right)_{3}\right]^{3-}$ (page 200) that, "contrary to earlier suggestions that all twelve oxygen atoms exchange, a thorough investigation has shown that only six exchange with solvent oxygen". This implies that the earlier report is wrong. That work was done at a higher temperature, however, and, furthermore, the experimental graphs in the second report show that, even at the lower temperature used, slow exchange beyond six oxygens does occur. The two reports surely reflect the existence of two routes for exchange.

Apart from the last chapter on organometallic systems, which includes an interesting survey of new reactions, mechanism and kinetics (in spite of a disclaimer) are treated as synonymous. If we instead agreed with Nyholm that kinetics : mechanism = fact : fiction, and if we further accept that this year's science fiction may be next year's frontier of research, future volumes of this series might usefully redress the balance between kinetic and other aspects of mechanism.

R. D. Gillard

\section{Artificial Intelligence}

Artificial Intelligence and Heuristic Programming. Edited by N. V. Findler and Bernard Meltzer. Pp. 327. (Edinburgh University: Edinburgh, August 1971.) £6.

THE first NATO Advanced Study Institute on Artificial Intelligence and Heuristic Programming was held in Italy in August 1970. Fourteen leading artificial intelligence (AI) research workers were invited to lecture on the states of the art and science of AI to an audience consisting mostly of informed students. This book contains a set of papers, based on those lectures. A compre- hensive index is included in the book. Collectively, the papers embrace most of the major themes in current AI research-computational logic, problem solving and problem solving compilers, natural language processing and models of cognitive processing. The papers are well written and as a book cohere through the noticeable consistency with which individual authors have balanced the tutorial and research-report content in their papers, due no doubt, to the editorial effect of the audience in the summer school.

The paper by Robinson on building deduction machines, and related remarks in the paper by Raphael on the "frame problem", contain perhaps the clearest statements of these topical issues to appear in the literature. Robinson's paper ends with remarks about the need to find upper and lower bounds on the amount of work required to carry out specified tasks, a theme related to Meltzer's discussion of efficiency in proof procedures. Needless to say, we are a long way from being able to formalize efficiency in even the simplest of tasks. In fact, it is doubtful whether at present we can indicate how a calculus of efficiency (which necessarily must work over some procedure specification) might be formulated. Meltzer's contribution to this important issue embraces a framework for examining proof procedures which he uses to sharpen the relationships among incompleteness, completeness and heuristic. $\mathrm{He}$ gives a lucid account of proof procedures as composites of inference systems and search strategies and pinpoints the intrusions of appeals to human performance.

At the other end of the spectrum of concepts which motivate AI work, Kochen, in a wide ranging paper, discusses cognitive learning processes. $\mathrm{He}$ rejects the current trends in AI towards better heuristics, powerful deductive inference schemes and so on, as being an entirely inappropriate path to an understanding of cognitive learning. He proposes and argues for a model which allows him to order hierarchically what could be meant by knowledge, utility, comprehension and wisdom and within which specificity is lent to psychological terms such as understanding, awareness, intention, thought and so on. This rather broad philosophically oriented exposition is supported by a mathematical model and some suggestive implementation results.

The natural language area is covered by contributions from Lindsay, Simmons and Palme. In Lindsay's paper we find another extremely clear statement of an important AI concept-the influence of context on computation. Lindsay reports on the design of a natural language learning system based in part on this principle. Palme takes 